\title{
Topological Anomaly Detection Performance with Multispectral Polarimetric Imagery
}

\author{
M.G. Gartley, W. Basener \\ Digital Imaging and Remote Sensing Laboratory \\ Chester F. Carlson Center for Imaging Science \\ Rochester Institute of Technology \\ 54 Lomb Memorial Drive \\ Rochester, NY 14623-5604
}

\begin{abstract}
Polarimetric imaging has demonstrated utility for increasing contrast of manmade targets above natural background clutter. Manual detection of manmade targets in multispectral polarimetric imagery can be challenging and a subjective process for large datasets. Analyst exploitation may be improved utilizing conventional anomaly detection algorithms such as RX. In this paper we examine the performance of a relatively new approach to anomaly detection, which leverages topology theory, applied to spectral polarimetric imagery. Detection results for manmade targets embedded in a complex natural background will be presented for both the RX and Topological Anomaly Detection (TAD) approaches. We will also present detailed results examining detection sensitivities relative to: (1) the number of spectral bands, (2) utilization of Stoke's images versus intensity images, and (3) airborne versus spaceborne measurements.
\end{abstract}

Keywords: Spectral, polarization, remote sensing, image simulation, scene simulation, BRDF, target detection, topology

\section{INTRODUCTION}

Polarimetric imaging (PI) has shown utility for helping solve a variety of specialized remote sensing problems. Although the general utility of PI appears to remain an interesting matter of debate, there is no doubt the modality is useful for the problem of locating man-made objects in cluttered backgrounds within the context of a large dataset when tasked appropriately. Conventional anomaly detection algorithms, notably $\mathrm{RX},{ }^{1}$ are commonly utilized on PI imagery to cue the user to areas of interest for further exploitation. However, one might ask whether PI adds value beyond a simple multispectral (MS) dataset which can provide similar cueing ability to an analyst when anomaly detection algorithms are applied.

Whether utilizing existing capability or designing new capability, the question of imaging targets with either MS, PI, or both is quite relevant. The answer to the question is obviously dependent on target material properties, acquisition geometry constraints, and background properties. In this paper we attempt to examine this problem in the context of trying to locate man-made targets in a background of dense foliage and shadows. More specifically, painted calibration panels and missile launchers are placed in various levels cover within the context of a suburban scene. We chose the Digital Imaging and Remote Sensing Image Generation (DIRSIG) model as a means to simulate remotely sensed spectral-polarimetric imagery from a variety of altitudes to perform a series of trade studies.

In an attempt to autonomously find the targets of interest in simulated spectral and/or polarimetric imagery we utilized the $\mathrm{RX}^{1}$ and $\mathrm{TAD}^{2}$ anomaly detection algorithms. The TAD algorithm is a new approach to anomaly detection and has been shown to exceed the performance of legacy algorithms such as RX on hyperspectral datasets. ${ }^{2}$ Through a series of trade studies, we examine the utility of unpolarized spectral, broadband polarimetric, and spectral polarimetric datasets of the same scenario utilizing both the TAD and RX anomaly detection algorithms. In addition, we examine the utility of performing anomaly detection on both the Stoke's polarimetric bands and the lower-level intensity sensed bands (the intensity bands are typically what is directly sensed, while the Stoke's images are merely a linear combination of these).

\footnotetext{
Algorithms and Technologies for Multispectral, Hyperspectral, and Ultraspectral Imagery XV, edited by Sylvia S. Shen, Paul E. Lewis, Proc. of SPIE Vol. 7334, 733410 · C 2009 SPIE CCC code: 0277-786X/09/\$18 - doi: 10.1117/12.817843
} 


\section{BACKGROUND}

Ideally this research would be dealing with imagery acquired from a real platform with unlimited ground truth. However, the nature of the trades being considered necessitate the use of simulated imagery. We wish to examine a notional collection platform that is similar in nature to commercial payloads on IKONOS or Quickbird retrofitted with linear polarizers to acquire 4 spectral bands and 4 linear polarization states per spectral band for a total of 16 bands. The resulting image datasets can be downsampled either spectrally and/or polarimetrically (discarding polarization information) to simulate many payload variations. In addition, the use of synthetic imagery permits extensive ground truth (exact locations of targets of interest, exact contribution of atmospheric path radiance etc.) which is essential for evaluation of the anomaly detection algorithms and understanding the phenomenology contributing to the results of the trade studies.

\subsection{Simulation of Spectral-Polarimetric Datasets}

The Digital Imaging and Remote Sensing Image Generation (DIRSIG) model is a complex synthetic image generation application which produces simulated imagery in the visible through thermal infrared regions. The model is designed to produce broad-band, multi-spectral and hyper-spectral imagery through the integration of a suite of first principles based radiation propagation sub models. These sub models are responsible for tasks ranging from the bi-directional reflectance distribution function (BRDF) predictions of a surface to the dynamic scanning geometry of a line scanning imaging instrument. In addition to sub models that have been specifically created for the DIRSIG model, several of these components (MODTRAN and FASCODE) are the modeling workhorses for the multi- and hyper-spectral community. All modeled components are combined using a spectral representation and integrated radiance images can be simultaneously produced for an arbitrary number of user defined bandpasses. Recently, the DIRSIG model has been upgraded to perform low-level radiometry (both reflective and emissive ${ }^{3}$ ) using spectral Stoke's and spectral Mueller matrix calculus to support simulation of polarimetric imaging platforms.

\subsubsection{Polarized Atmosphere}

DIRSIG leverages the experimental MODTRAN4-P radiative transfer code to simulate the polarimetric radiance of the skydome and the polarized solar-scattered path radiance between the ground and the sensor. The integration of MODTRAN-P with the DIRSIG model has been verified against community accepted sky polarization Coulson tables. ${ }^{4}$ Consideration of the sky polarization is key to accurate polarimetric image simulations due to the dominance of sky radiance on objects in shadows and the potential for a strong polarimetric bias in the sensor to target path radiance which may have a noticeable effect on target detection algorithm performance.

\subsubsection{Polarimetric Material Properties}

Another key consideration of the simulation environment is the accurate representation of the polarimetric scattering properties of both targets and backgrounds. For man-made target materials we have leveraged a combination of experimentally measured polarimetric bi-directional reflectance distribution function (pBRDF) data fit to analytical models and pBRDF parameters from the Non-conventional Exploitation Factors database. ${ }^{5}$ For some of the background materials we leverage existing clutter pBRDF models. ${ }^{6}$

One example of a material we have experimentally measured the pBRDF for at RIT is roofing shingles. We have found the polarimetric signature coming from various colors of roofing shingles (from the same manufacturer) tend to be very similar, however the unpolarized component of the pBRDF of the shingles carries the majority of the spectral shape. In addition, we have characterized vinyl siding in the lab and found similar behavior. For other man-made materials, we are able to leverage the Beard-Maxwell pBRDF parameters from the NEF database. In addition to laboratory measurements, RIT has also characterized a variety of background materials (such as grass, asphalt and dirt) in the field and fit a parametric Roujean-based pBRDF model to the results.

One background material that is particularly difficult to characterize polarimetrically with lab-based or ground-based equipment is trees. In order to develop confidence in a tree polarization model, we made a small set of lab measurements on single leaf surfaces and verified the pBRDF properties against published results. ${ }^{7}$ However, in order to determine whether the simulation of the leaf pBRDF properties in the context of a forest was accurate, we utilized a variant of DIRSIG to simulate the pBRDF of a forest (planted with trees having 
the measured leaf pBRDF and unpolarized trunks and branches) and verified the degree of linear polarization (DOLP) results against publicly available POLDER measurements of broadleaf forest species. ${ }^{8,9}$

\subsection{Anomaly Detection Algorithms}

Finding anomalies in large data sets is an important problem in many applications, particularly in technological applications involving enormous amounts of data where the first step is often to filter out the background noise and highlight the most important information. For example, credit card issuers need to flag unusual spending patterns for the purpose of detecting theft. Detecting underground structures with unusual chemical composition, texture and density, called geo-anomalies, is important for both determining the genesis of the geology as well as detection of natural resources.1 Anomaly detection is an essential process in the image analysis and in remote sensing.

\subsubsection{RX}

One straightforward algorithm for anomaly detection is to compute the mean of the data, and define the anomalies to be the points that are farthest from that mean. This is the idea behind the RX algorithm, ${ }^{1}$ which is commonly found in image processing software packages. The covariance matrix for the data from the annular is computed, and the rank of each pixel becomes the number of standard deviations from the mean of the data from the data. Typically, there will be a small number of directions in which the covariance is large and the covariance will be very small in the remaining directions. Hence, the ellipsoids will be pancake-shaped.

The RX algorithm works well for some anomalies in some situations. However, since the RX algorithm uses a covariance, there is an inherent assumption of normality in the data, which typically is not the case for hyperspectral and/or polarimetric imagery. Anomalies can hide within the ellipsoids despite being far away from most other data points. This is due to the complex nonlinear geometry of the data.

\subsubsection{TAD}

Let $X$ be a finite collection of points in $R^{k}$. Typically, we think of $X$ as having on the order of one million points and k around 200. Let $r$ be a positive real number. Define $G_{r}$ to be the graph whose vertex set is $X$ such that there is an edge from $x$ to $y$ iff the distance from $x$ to $y$ is less than $r$. We call $G_{r}$ to be the graph of resolution $r$ for the data set $X$. We call $r$ the resolution of the graph, since we think of two points of distance less than $r$ as being indistinguishable. We can also think of $G_{r}$ as the graph obtained by placing balls of radius $r$ around every point and connecting $x$ and $y$ with a vertex if the ball of radius $r$ around $x$ contains $y$.

In constructing $G_{r}$, we think of two points $x$ and $y$ as being similar if the distance between the points is less than our resolution $r$. In the case of hyperspectral imagery, the distance between $x$ and $y$ is less than $r$ if the pixels in the image for those points represent similar materials on the ground. Thus, we care most about the components of $G_{r}$, since two points in the same component should have similar material.

We think of the points in large components of $G_{r}$ as background points, and all nonbackground points as being an anomalies. Specifically, let $p$ be a percentage between 0 and 100, which we will call the background percentage. Define a component $H$ of $G_{r}$ to be a background component if $H$ contains more than $p$ of the points of $X$. A point in a background component is called a background point, and a point that is not in a background component is called an anomaly. In practice, we expect to choose $p$ to be approximately $1 \%$ and we expect roughly $95 \%$ of the points in $\mathrm{X}$ to be background points although this can vary by application. We now define the degree of an anomaly $x$ to be the distance from $x$ to the set of background points, which is the minimum of $\mathrm{d}(\mathrm{x}, \mathrm{y}) \mid \mathrm{y}$ is a background point, or, equivalently, the distance from $x$ to the nearest background point. Anomaly detection algorithms use the data to create an anomaly function from spectral space $R^{k}$ to $R_{+}$, which we call the rank function. The algorithm then evaluates the rank function at the location of each data point to determine how anomalous they are. 


\section{APPROACH}

The experimental approach for this work consists of generating high spatial and spectral resolution spectralpolarimetric datasets utilizing DIRSIG. These high resolution datasets were 1024x1024 pixels in spatial size, spectrally resolved from 0.4 to 1.0 into $50 \mathrm{~nm}$ bands, and polarimetrically resolved into all four Stoke's parameters S0, S1, S2, and S3. The high resolution datasets were generated utilizing collection platforms at 5, 50, and 500km above the ground with the same effective look angle and ground resolution. The sensor elevation angle was 46 degrees with a relative azimuth to the sun of 151 degrees (where 180 degrees is the solar forward scattering direction). The solar elevation angle was 12 degrees above the horizon. The various trades were performed by downsampling the high resolution data in spatial extent (to reduce aliasing resulting from the simulation) and spectral extent as needed.

\subsection{Simulated Collection Platforms}

For this work, we assumed a notional variant of the commercial IKONOS collection platform. This variant has exactly the same 4 spectral bands (blue, green, red, and near-IR), except each band is collected polarimetrically. The normalized spectral responses ${ }^{10}$ of each spectral band are shown in Figure 3. To collect polarization information, each spectral band is fit with a combination of 4 linear polarizers (oriented at 0, 45, 90, and 135 relative to the telescope boresight) designed to sense the linear polarization state of the radiance reaching the telescope.

\subsection{Synthetic Scene}

The synthetic scene utilized for this study was a small portion of the DIRSIG Megascene 1 (Figure 1a), which represent a portion of a suburb to the north of the Rochester Institute of Technology. Extensive ground truth (DEM, spectral measurements, geolocation of objects) has been leveraged to create a $1 \mathrm{~km} \times 3 \mathrm{~km}$ scene that is frequently utilized for hyperspectral image simulations. A $0.4 \mathrm{~km}$ x $0.4 \mathrm{~km}$ subset of Megascene 1 was chosen for this work because of its combination of dense foliage, a grassy clearing and many houses and roads which would provide a representative level of spectral and polarimetric background clutter for detection of man-made objects.

The targets of interest that were placed in the scene were 5 metal missile launchers (at various orientations) in the tree shadows, one metal missile launcher in the sun next to a house, and 2 CARC green painted calibration panels positioned in the clearing in clear view of the sun (see Figure 1b). The CARC green calibration panels have a moderate polarization signature, and a spectral appearance similar to the grass in the visible portion of spectrum. All six missile launchers were attributed with a moderately shiny metal leveraged from the NEF database, for which the reflectance polarization properties have been verified. Although the polarization signature of the launcher material would typically be more significant compared to the CARC green paint, the geometric complexity of the launcher and the placement of them renders them somewhat difficult to isolate both spectrally and polarimetrically.

The following 6 synthetic datasets were generated for the 5,50 , and $500 \mathrm{~km}$ altitude platforms, for a total of 18 unique datasets:

1. Multispectral polarimetric: 4 polarimetric intensity bands (I0, I45, I90, I135) x 4 spectral bands

2. Multispectral polarimetric: 3 Stoke's bands (S0 ,S1, S2) x 4 spectral bands

3. Multispectral polarimetric: 2 Stoke's bands (S1, S2) x 4 spectral bands

4. Panchromatic polarimetric: 4 polarimetric intensity bands (I0, I45, I90, I135)

5. Panchromatic polarimetric: 3 Stoke's bands (S0, S1, S2))

6. Multispectral (4 spectral bands)

In addition, other variants of this notional platform were considered for use in the trade studies. One variant consisted of one spectrally broad (0.4 - 1.0 microns) polarimetric band collected polarimetrically (I0, I45, I90, and I135). Another variant was simply the standard IKONOS platform, which has 4 bands and is polarimetric. 


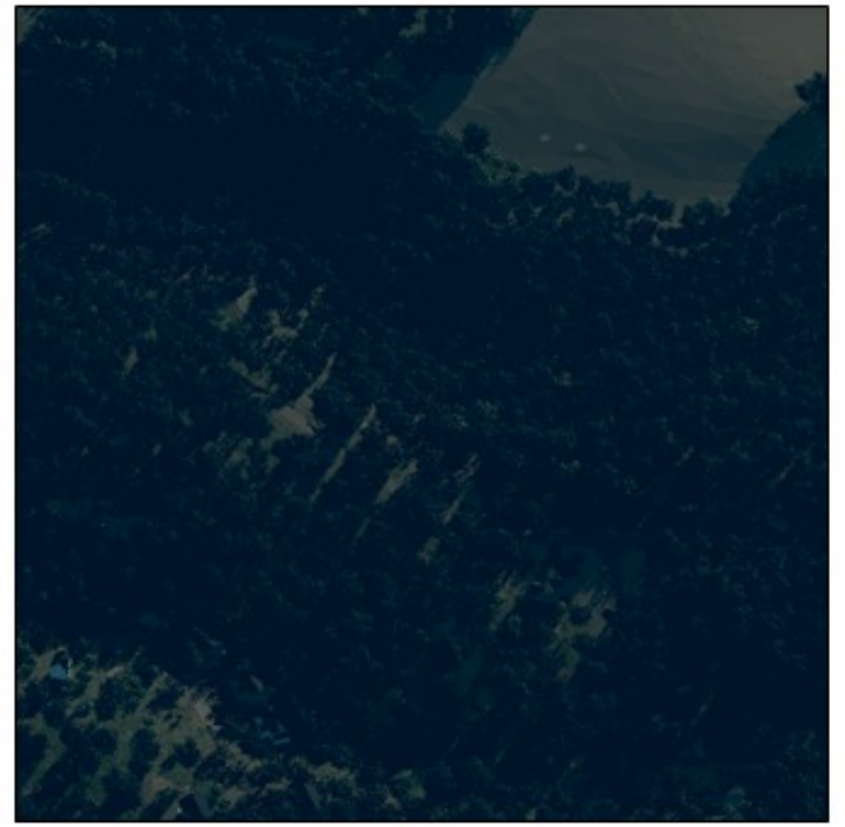

(a)

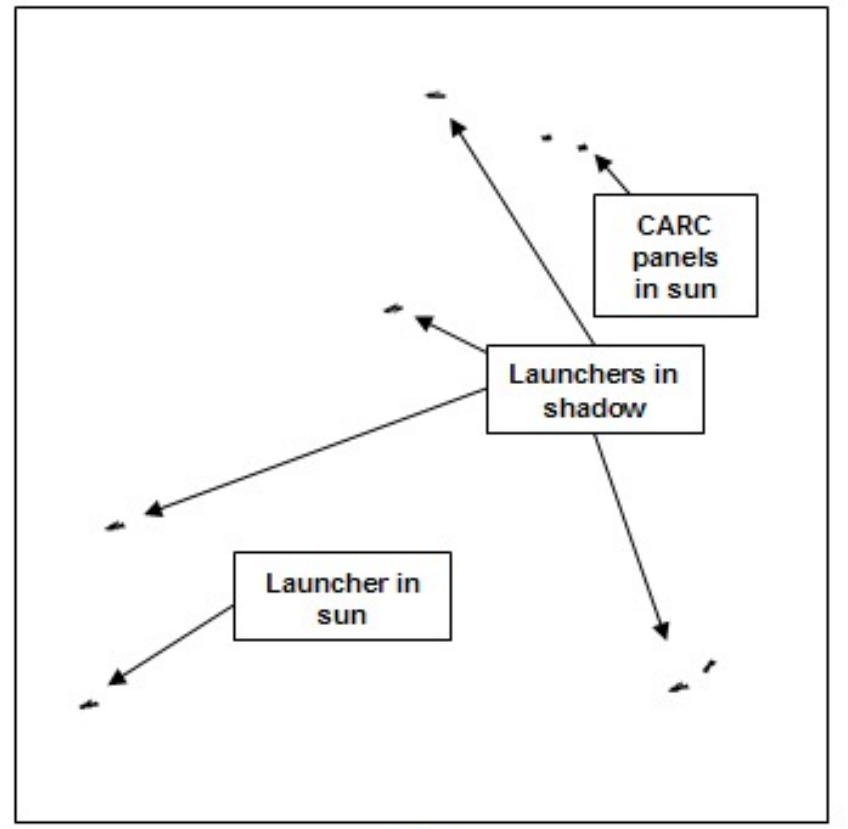

(b)

Figure 1. Synthetic scene used for this research containing a CARC green panel targets and missle launchers in a background of broadleaf forest, grass, roads, and homes.

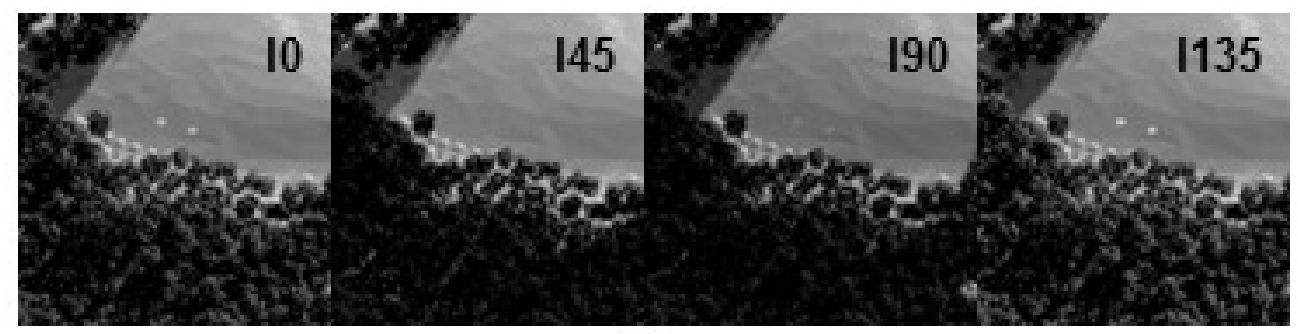

(a)

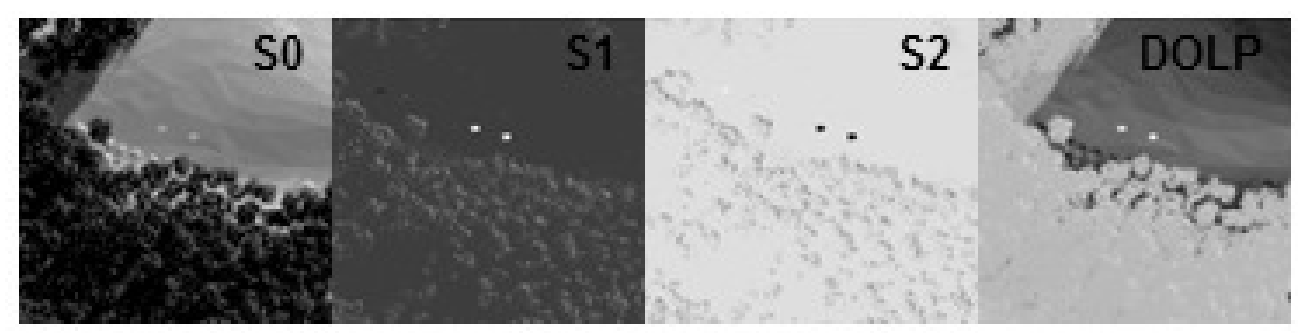

(b)

Figure 2. A chip from the simulated scene showing (a) the four collected broadband intensity images and (b) the derived Stoke's and DOLP images. 


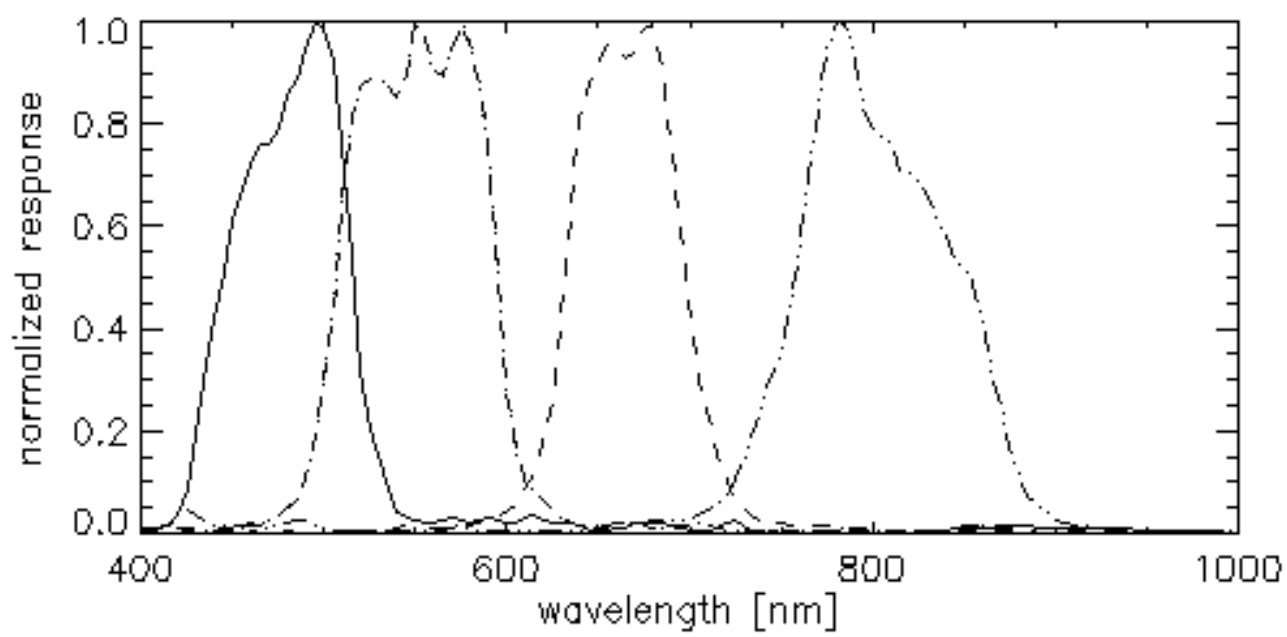

Figure 3. Normalized plots of the spectral response functions for the commercial IKONOS multispectral payload.

\section{RESULTS}

A common method for quantifying the efficacy of an anomaly detection algorithm is by plotting a receiver operating characteristic (ROC) curve. This curve relates the target detection rate as a function of the false alarm rate. We normalize the detection rate to be equal to 1.0 when all targets have been found. In the case of this study, the targets of interest are the five missile launchers in shadow, the one launcher in the sun next to the house, and the two CARC green panels in the sun on the grass clearing. The truth mask (Figure 1b) was downsampled $2 \mathrm{x}$ (similar to the original radiance image cube) resulting in a floating point truth image. A value of 1.0 indicates the pixel is $100 \%$ target, while a value of 0.25 indicates the pixel is only $25 \%$ target. All pixels with a truth mask value of 0.5 (50\% target) or greater were considered target pixels, while all others were considered non-target background pixels for ROC curve generation.

\subsection{Target Detection in Stoke's vs. Intensity Imagery}

The first trade examines the performance of both the TAD and RX algorithms at finding the six missile launchers and two painted panels in the complex spectral-polarimetric background clutter. The left and middle columns of Figure 4 present the ROC curves for 16 band multispectral polarimetric (intensity) and 12 band multispectral polarimetric Stoke's images. Recall that the Stoke's images are not typically directly sensed, but merely a linear combination of the directly sensed intensity bands (I0, I45, I90, and I135). These results show the TAD algorithm performs exactly the same regardless of whether the 16 intensity or 12 Stoke's image bands are utilized. However, the RX algorithm appears to take a significant performance hit (actually doing worse than simply 50/50 guessing!) when operating on the intensity image bands compared to the Stoke's image bands where it performs quite well.

As a confirmation of these results, we find a similar behavior for algorithm performance when operating on panchromatic intensity vs. Stoke's imagery. The TAD algorithm ROC curve is identical whether it operates on the intensity or Stoke's image bands, while the RX algorithm performs poorly when applied to the four intensity image bands relative to application on the three Stoke's bands.

\subsection{Target Detection on Airborne vs. Spaceborne Collected Imagery}

The next trade examines the effect of the effect of the upwelled sky polarization on the anomaly algorithm detection performance. For space borne platforms, the field of view across the entire image is quite small as is the relative difference in the upwelled Stoke's radiance across the entire image. In this case, the effect of the sky polarization is essentially a spatially constant bias across the image extent. However, the airborne collected imagery is subjected to a larger variation in the upwelled sky radiance across the image plane due to a much larger field of view. This trade is meant to examine the effect of the sky polarization, and it's spatial distribution across the image, on target detection performance. 


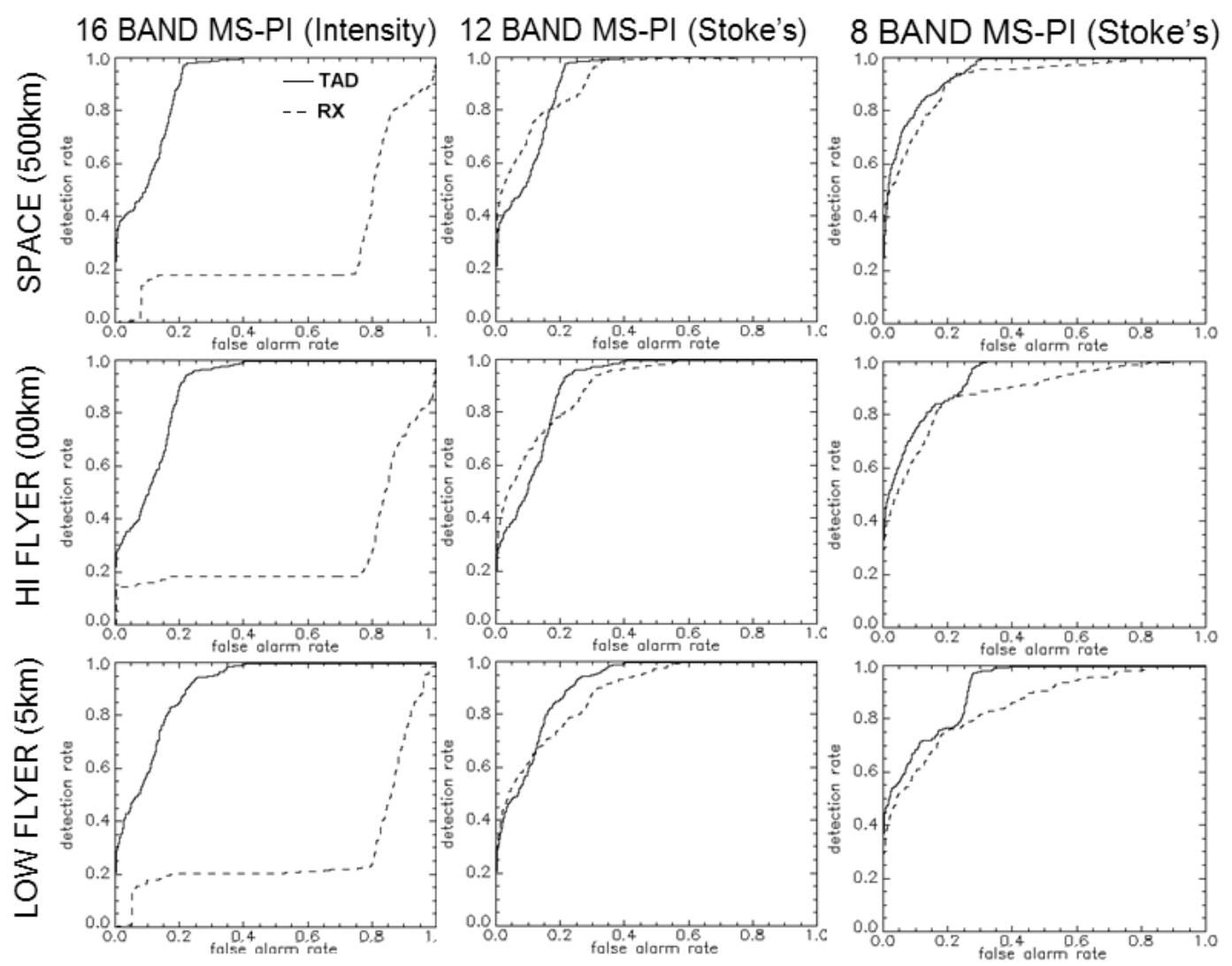

Figure 4. Receiver operating characteristic curves for locating man-made objects with unique spectral-polarimetric reflectance properties for a low flying (top row), high flying airborne platform (middle row), and a space borne platform (bottom row). The solid lines represent the TAD algorithm performance, while the dotted lines represent the global Rx algorithm performance on 16 band multispectral polarimetric-intensity (left column), 12 band multispectral polarimetric Stoke's (middle column) and 8 band multispectral Stoke's images (right column).

Review of the top rows of Figures 4 and 5 show the performance for different combinations of spectral and polarimetric imagery bands collected from space borne platform. The performances on the same datasets, except collected from a low flying airborne platform, are shown in the bottom rows of Figures 4 and 5. In all six cases, the RX algorithm performed better on the space borne platform collected imagery compared to the low flying airborne collected imagery. The TAD algorithm appears to be slightly more invariant to the collection altitude, exhibiting slight performance gains at higher altitudes. However the TAD algorithm doesn't appear to be as sensitive to the magnitude and spatial variation of the upwelled radiance as the RX algorithm is. In other words, the RX algorithm appears to be more sensitive to a non-uniform, low-frequency bias while the TAD algorithm is slightly more robust.

\subsection{Target Detection on Multispectral vs. Polarimetric Imagery}

Considering the complexity of a 16 band $(4$ spectral x 4 polarimetric $=16)$ collection payload, another interesting trade is to examine the target detection performance of both algorithms on limited band datasets, namely panchromatic polarimetric vs. unpolarized multispectral imagery (requiring only 4 bands each). For this trade, we examine the plots in Figure 5 that demonstrate a distinct performance advantage for the RX algorithm operating in the multispectral domain vs. the broadband polarimetric domain. However, interestingly enough the opposite appears true for the TAD algorithm. More specifically, the TAD algorithm is able to find the targets of interest more rapidly in the broadband polarimetric imagery compared to the unpolarized multispectral imagery. Inspection of the scatter plots of S1 versus S2 show the targets buried deeper into the polarimetric clutter (in a non-Gaussian fashion) which is likely what makes it more difficult for RX to find them. 

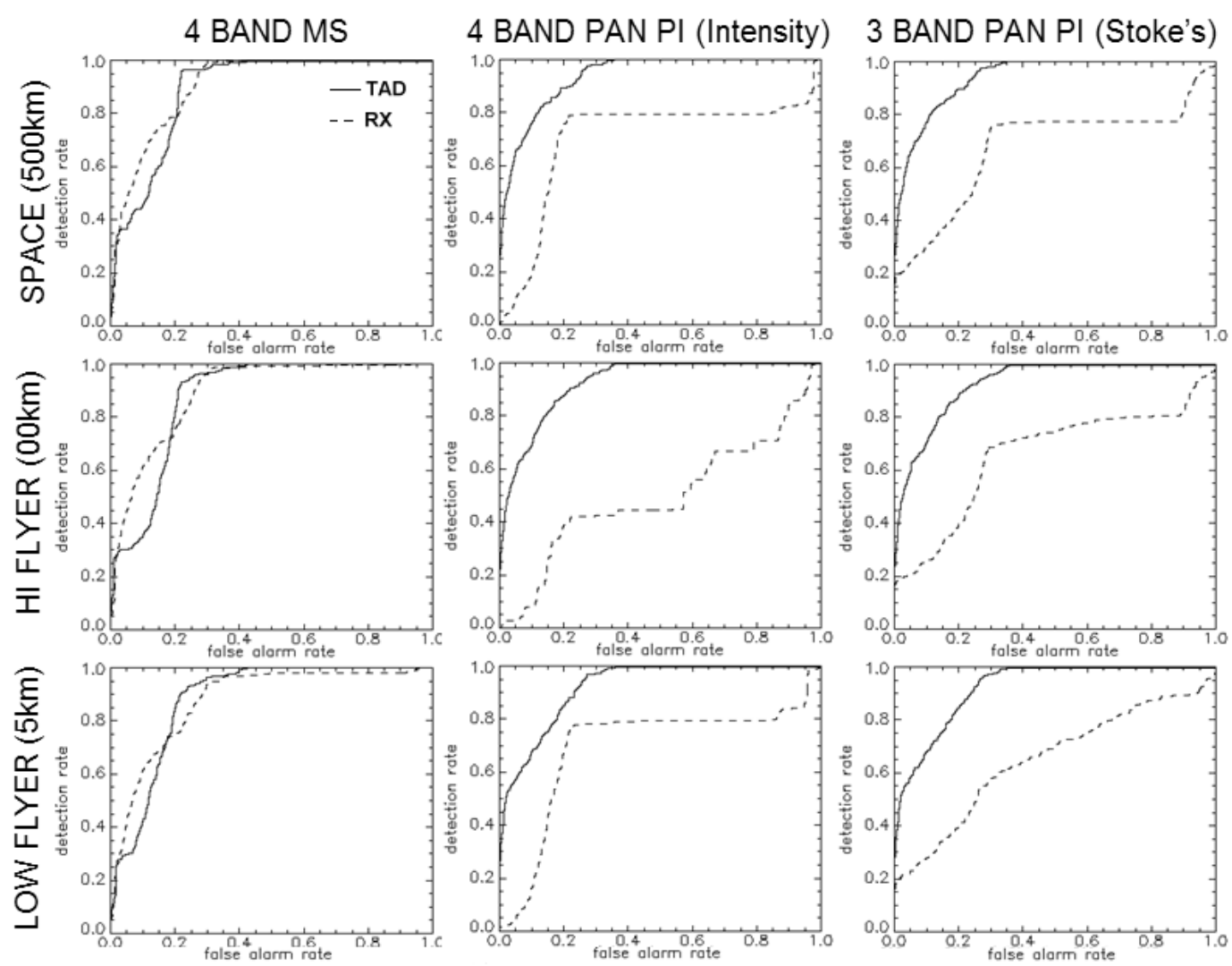

Figure 5. Receiver operating characteristic curves for locating man-made objects with unique spectral-polarimetric reflectance properties for a low flying (top row), high flying airborne platform (middle row), and a space borne platform (bottom row). The solid lines represent the TAD algorithm performance, while the dotted lines represent the global Rx algorithm performance on 4 band multispectral (left column), 4 band panchromatic polarimetric intensity (middle column) and 3 panchromatic Stoke's images (right column).

\subsection{Target Detection via RX vs. TAD}

Comparison of the RX algorithm against the TAD algorithm for finding the man made targets of interest in the synthetic spectral polarimetric scene shows mixed results. As stated earlier, the RX algorithm appeared to have degraded performance when operating on polarimetric intensity images versus polarimetric Stoke's images, while the TAD algorithm's performance was invariant to this band selection. This feature of the TAD algorithm appears to be a result of the linear, shift-invariant feature of it's operation.

Similar to previous findings ${ }^{2}$ showing the TAD algorithm outperforming RX on hyperspectral datasets, we find that TAD does equally well or better for each of the 18 spectral-polarimetric datasets of this work. In addition to meeting or exceeding RX performance, we also present the visualization ability of the TAD results via a color display of the first three principal components decomposition bands. Figure 6 shows an example of the RX output and the TAD algorithm output (with a principal components visualization output) as applied to the low flying airborne collection using 12 spectral Stoke's image bands. The TAD visualization not only detects more of the target edge pixels, but actually discriminates the targets into classes by color. The missile launchers (both in shadow and sun) have a blue tone compared to the CARC green painted panels which have a strong green tone. The visualization clearly aids in classifying the anomalous pixels.

In addition, we find that when the TAD algorithm is restricted to seeing only S1 and S2 polarimetric bands, the performance is slightly better than when all three Stoke's bands are analyzed (compare the right column and middle columns of Figure 4). This performance boost for TAD is not surprising given that the targets of interest are known to have a polarimetric reflectance behavior, whereas the background pixels are a mix of polarized and 

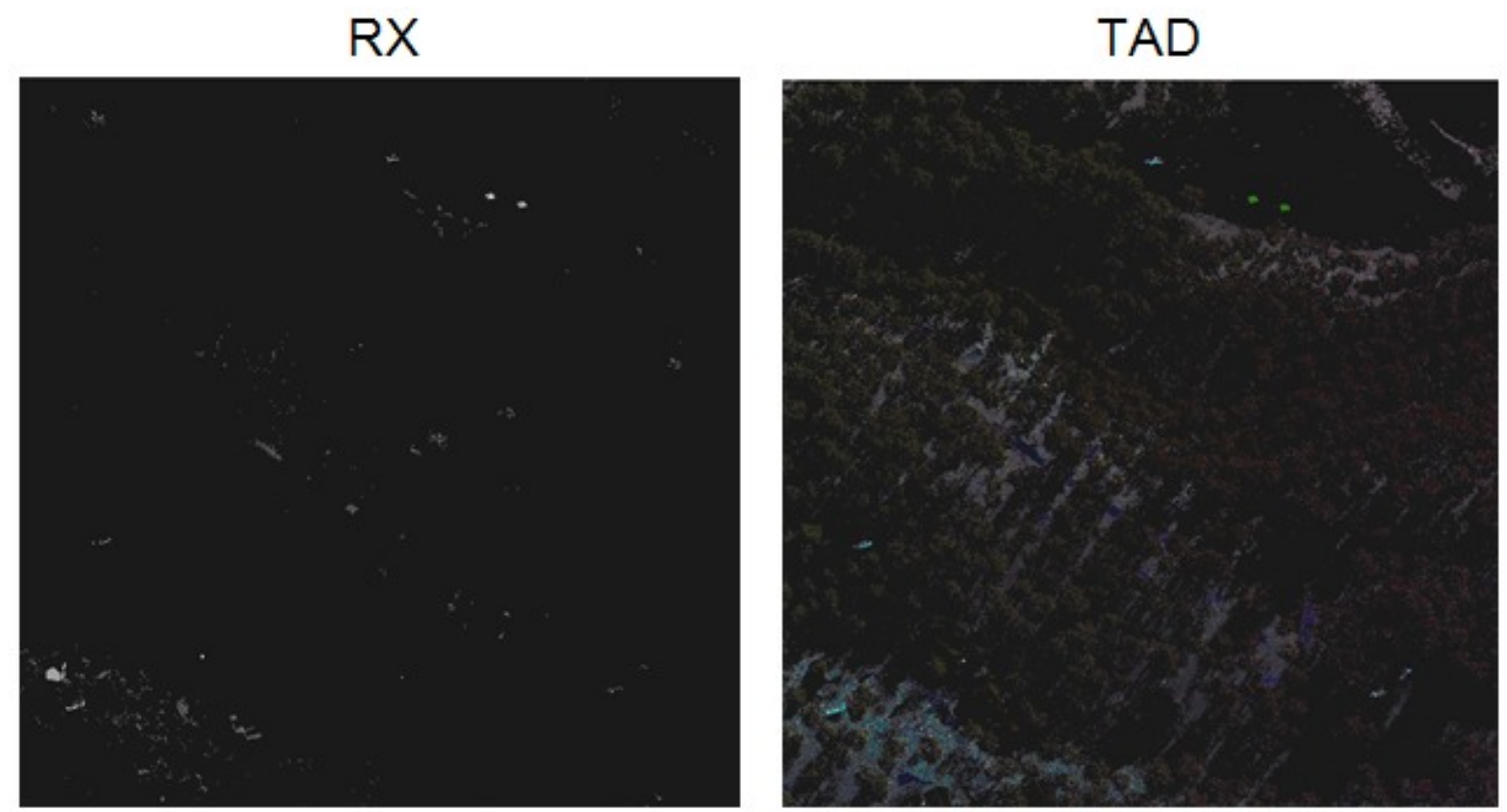

Figure 6. Comparison of the RX algorithm output (left) with a color visualization of the principal components decomposition output from TAD (right).

un-polarized reflectances, and the algorithm is better able to discriminate between background and target pixels.

Finally, we find that in all 18 dataset cases the TAD algorithm does a better job of locating target edge pixels relative to the RX algorithm. Although detection of each and every edge pixel is not a requirement of most anomaly detection tasks, it does indicate that TAD might perform equally well under stressing MTF (heavy levels of image blur) and under-resolved sampling (sub-pixel targets) conditions.

\subsection{Detection Sensitivity Considering MTF and Noise Effects}

The previous trades have generated some understanding as to the performance of both the TAD and RX algorithms for detecting man-made objects in shadow and sun as a function of collection altitude and the number of spectral and polarimetric bands collected under pristine conditions. However, a more realistic scenario is to apply the effects of both image blur and noise. The image blur results from the optical diffraction modulation transfer function (MTF), the focal plane MTF, and scanning/platform effects MTF (such as scanning motion, jitter). For simplicity, we approximate the notional IKONOS payload as having a $\lambda F N / p^{11}$ value of 0.8 and a half pixel smear due to platform motion. The noise in the sensed image originates from the sensor reaching radiance photon shot noise as well as sensor level noise. For this trade, we simply added a Gaussian distributed noise term to the raw intensity (I0, I45, I90, I135) images. Two versions of the platform were considered here: (1) a space based and broadband polarimetric and (2) a low flying airborne 16 band MS-PI platform. The resulting Stoke's S1, and S2 images therefore have increased noise due to the differencing of intensity bands, while the derived S0 image has reduced noise due to the co-addition of intensity bands. No band to band mis-registration was modeled for this case.

Figure 7 shows the broadband polarimetric Stoke's images for SNR levels of 5000, 15 and 5 . The SNR values are the average SNR calculated in the intensity bands at $550 \mathrm{~nm}$ for the grassy clearing relative to the dark shadowed areas. Consistent with the results from a previous section, we find the TAD algorithm to perform better at locating the targets of interest as anomalous, even in the presence of significant noise and MTF effects (see Figures 8 and 9). 


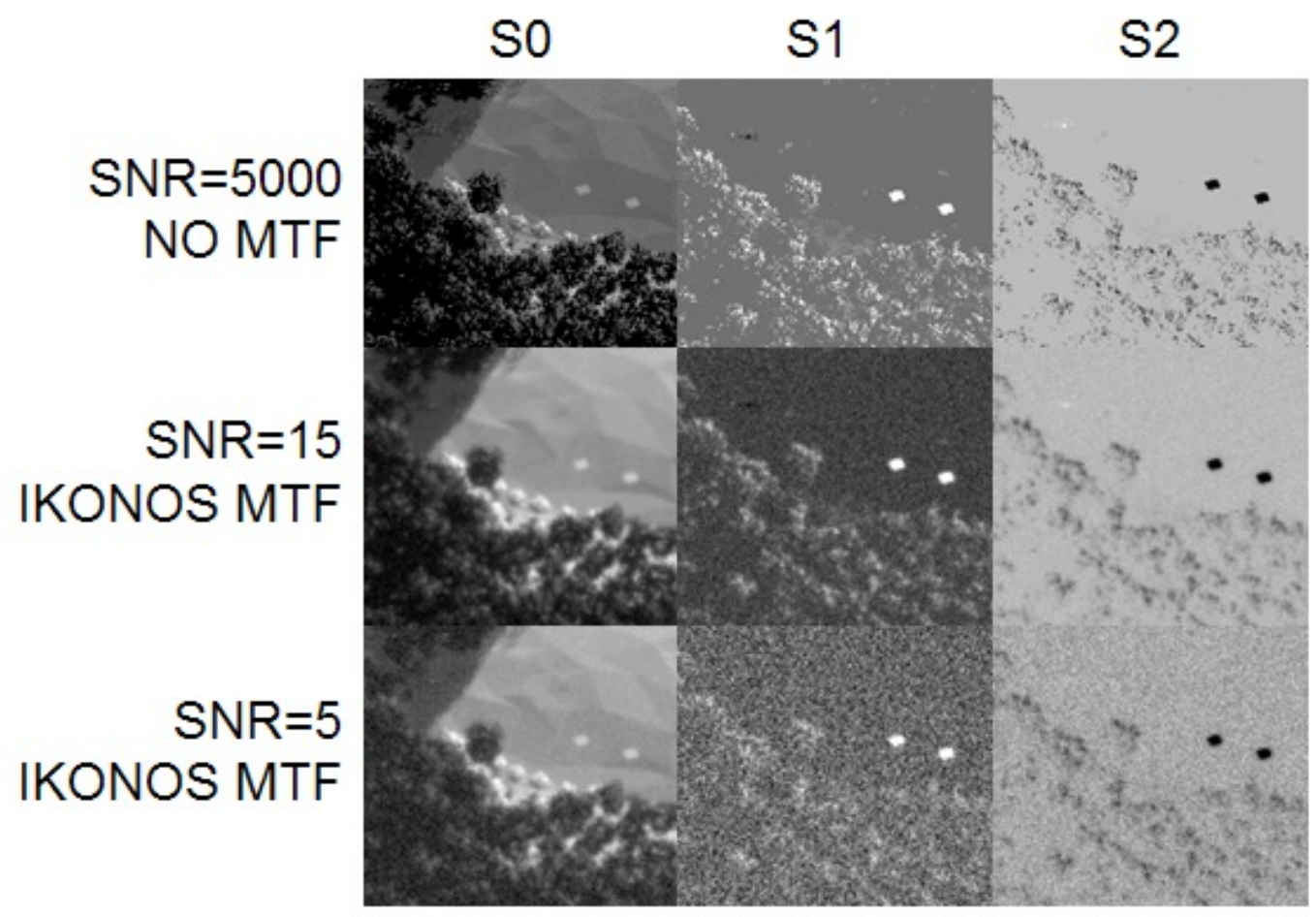

Figure 7. Panel of images showing the effect of MTF and noise (SNR is calculated on grass at 550nm in the directly sensed intensity bands) on derived broadband Stoke's image bands.

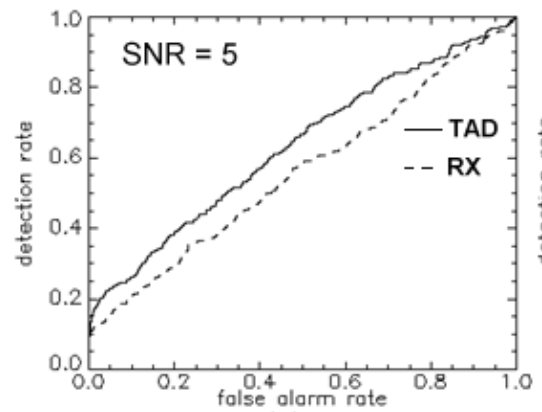

(a)

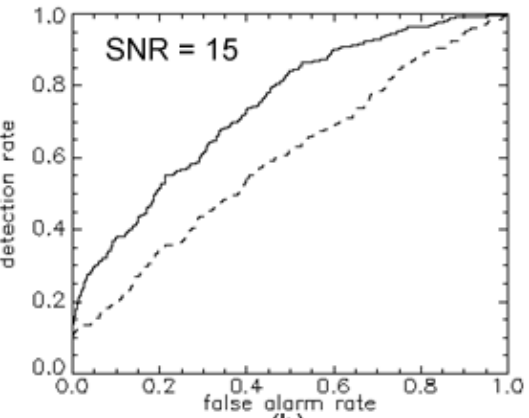

(b)

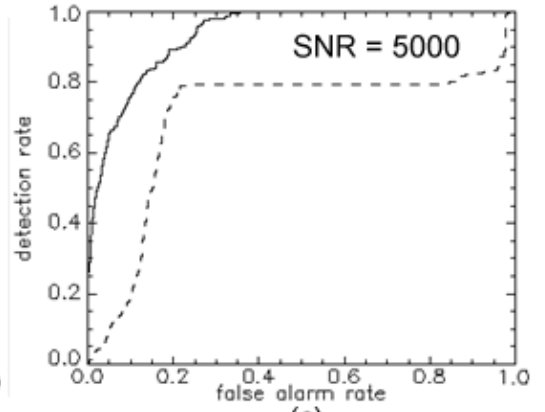

(c)

Figure 8. ROC curves showing the effect of noise and MTF on target detection performance for both TAD (solid line) and RX (dashed line), where (a) SNR $=5$ and MTF blur, (b) SNR $=15$ and MTF blur, and (c) SNR $=5000$ and no MTF blur for a broadband Stoke's image product (S0, S1, and S2) collected from a spaceborne platform. 


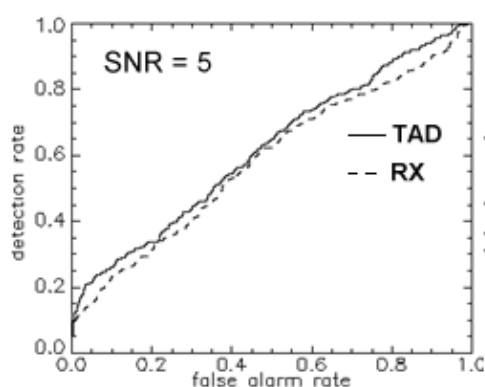

(a)

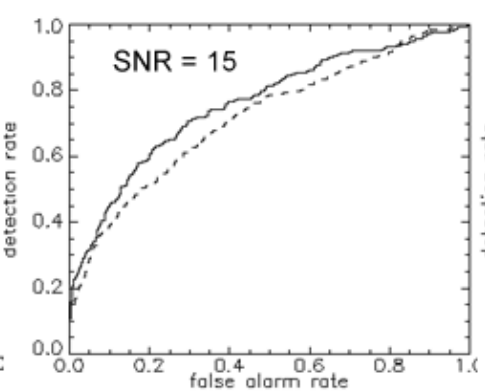

(b)

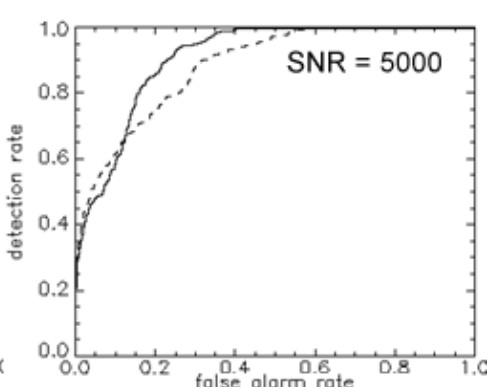

(c)

Figure 9. ROC curves showing the effect of noise and MTF on target detection performance for both TAD (solid line) and RX (dashed line), where (a) SNR $=5$ and MTF blur, (b) SNR = 15 and MTF blur, and (c) SNR = 5000 and no MTF blur for a 12 band (4 spectral bands x 3 Stoke's bands) image product collected from a low flying airborne platform.

\section{SUMMARY}

It comes as no surprise that we find no clear answer to what image modality and collection of spectral-polarimetric bands is better for finding man-made targets in a cluttered background, any more than it's no surprise that any one given anomaly detection algorithm is not better than another for all cases. However, our limited look at man-made objects located both in the shade and in the sun allow us to see trends between modalities, collection altitudes, and anomaly detection algorithms.

First, we find the upwelled sky radiance (both magnitude and spatial variability across the image plane) to have a measurable effect on target detection performance. The TAD algorithm appeared to be less sensitive to the effect of the upwelled radiance compared to the RX algorithm which performed consistently better on higher altitudes (where the spatial variability of the upwelled radiance is minimal) relative to lower altitudes (spatial variability of upwelled radiance is high). Secondly, we found the RX algorithm performance was enhanced by performing the detection in the Stoke's imagery vs. the raw intensity band imagery. However, the TAD algorithm performance was invariant to operating in either the intensity or Stoke's image domains. What's more, we find a TAD performance boost by eliminating the unpolarized Stoke's band and operating simply on the S1 and S2 image bands. whereas the RX algorithm faired worse by this S0 removal tactic. We also find with limited bands the RX algorithm outperforms the TAD algorithm on unpolarized multispectral imagery while the TAD algorithm outperforms RX on panchromatic polarimetric imagery. One important result of this work showed that in all 18 dataset cases the TAD algorithm does a better job of locating target edge pixels relative to the RX algorithm. Although detection of each and every edge pixel is not a requirement of most anomaly detection tasks, it does indicate that TAD might perform equally well under stressing MTF (heavy levels of image blur) and under-resolved sampling (sub-pixel targets) conditions.

We see the take away from this work to be that anomaly detection performance is highly dependent on payload configuration and the number of available polarized and unpolarized spectral bands. Future work will examine the same trades utilizing a more robust system and noise model, incorporating both MTF and shot noise effects on the trade study results. A preliminary system model was examined in this work for a subset of the trades and shown that the TAD algorithm continues to outperform RX under stressing MTF and noise conditions. With the rigorous incorporation of a polychromatic MTF and both sensor level and incident photon shot noise, we expect the Stoke's imagery to be significantly more noisy compared to the directly sensed intensity imagery (since the S1 and S2 Stoke's bands are differencing the intensity bands). In this case we expect the TAD algorithm to more strongly outperform RX by its ability to operate equally in either the Stoke's or intensity band space, which did not appear to be the case for RX in our work.

\section{ACKNOWLEDGMENTS}

Michael Gartley wishes to thank the Intelligence Community Post-Doctoral Fellowship Research program and Lockheed Martin Information Systems \& Global Services Enterprise Integration Group for supporting portions of this research. 


\section{REFERENCES}

1. I. Reed and X. Yo, "Adaptive multi-band cfar detection of an optical pattern with an unknown spectral distribution," IEEE Trans. Acoustics, Speech, and Signal Proc. 38, pp. 1760-1770, October 1990.

2. W. Basener, E. Ientilluci, and D. Messinger, "Anomaly detection using topology," Proc. of SPIE, Algorithms and Technologies for Multispectral, Hyperspectral, and Ultraspectral Imagery XIII 6565, 2007.

3. M. Gartley, S. Brown, and J. Schott, "Polarimetric scene modeling in the thermal infrared," Proc. of SPIE, Polarization Science and Remote Sensing III 6682, 2007.

4. C. Devaraj, S. Brown, D. Messinger, A. Goodenough, and D. Pogorzala, "A framework for polarized radiance signature prediction for natural scenes," Proc. of SPIE, Algorithms and Technologies for Multispectral, Hyperspectral, and Ultraspectral Imagery XIII 6565, 2007.

5. Nonconventional Exploitation Factors Data System (NEFDS) Modeling Document v10, National Geospatial Intelligence Agency, 2006.

6. J. R. S. II and J. R. Schott, "A polarized clutter measurement technique based on the governing equation for polarimetric remote sensing in the visible to near infrared," Targets and Backgrounds XI: Characterization and Representation 5811(1), pp. 34-45, SPIE, 2005.

7. L. Bousquet, S. Lachrade, S. Jacquemoud, and I. Moya, "Leaf brdf measurements and model for specular and diffuse components differentiation," Remote Sensing of Environment 98(2-3), pp. 201 - 211, 2005.

8. P.-Y. Deschamps, F.-M. Breon, M. Leroy, A. Podaire, A. Bricaud, J.-C. Buriez, and G. Seze, "The polder mission: instrument characteristics and scientific objectives," Geoscience and Remote Sensing, IEEE Transactions on 32, pp. 598-615, May 1994.

9. J. Chen, S. Leblanc, J. Cihlar, P. Bicheron, M. Leroy, D. Deering, and T. Eck, "Studies of brdf in conifer and deciduous boreal forests using the 4-scale model and airborne polder and ground-based parabola measurements," Geoscience and Remote Sensing, 1997. IGARSS '97. Remote Sensing - A Scientific Vision for Sustainable Development., 1997 IEEE International 1, pp. 165-167 vol.1, Aug 1997.

10. J.-I. Kudoh, K. Kalpoma, and Y. Kurita, "A process to minimize the spectral distortion in 1m ikonos color image by using four parameters," Geoscience and Remote Sensing Symposium, 2006. IGARSS 2006. IEEE International Conference on , pp. 3240-3243, 31 2006-Aug. 42006.

11. R. D. Fiete, "Image quality and lambda fn/p for remote sensing systems," Optical Engineering 38(7), pp. 1229-1240, 1999. 